УДК 66.048.3

РАСЧЕТ ПРОЦЕССА СОВМЕЩЕННОЙ КОНДЕНСАЦИИ И ИСПАРЕНИЯ МНОГОКОМПОНЕНТНОЙ СМЕСИ

\title{
PROCESS CALCULATION OF COMBINED CONDENSATION AND MULTICOMPONENT MIXTURE EVAPORATION
}

\author{
3.Ф. Мингалимов, Т.Г. Умергалин
}

Уфимский государственный нефтяной технический университет, г. Уфа, Российская Федерация

\section{Zagir F. Mingalimov, Talgat G. Umergalin}

\section{Ufa State Petroleum Technological University, Ufa, Russian Federation e-mail: mingalimov.z@gmail.com}

Аннотация. Совмещенный процесс многоступенчатой конденсации и испарения является простым и одним из эффективных способов разделения многокомпонентной смеси. Данный процесс может быть реализован в фракционирующем аппарате горизонтального исполнения. Теплоноситель (хладоноситель), использованный на предыдущей ступени испарения (конденсации), по ходу движения направляется для повторного использования в последующую ступень и т.д. Тем самым обеспечивается более полное снятие тепла (холода) теплоносителя (хладоносителя).

Анализ процесса совмещенной многоступенчатой конденсации и испарения смеси можно проводить с помощью графической комбинированной энтальпийной диаграммы, уравнений рабочей линии и кривой равновесия фаз, а также на основе других закономерностей, применяемых при анализе процесса ректификации. Графические методы не точны и годны лишь для анализа разделения бинарной смеси. Предпочтительны компьютерные расчеты, основанные на устойчивых 
итерационных методах. Ввиду существенной особенности процесса многоступенчатой совмещенной конденсации и испарения применение общеизвестных методов расчета разделения многокомпонентной смеси ректификацией не удается.

Применительно к данному процессу разработан итерационный метод расчета, основанный на смешении потоков пара и жидкости, вводимых в совмещенную ступень, решении системы нелинейных уравнений теплового, материального балансов и однократного испарения, определения величин образованных потоков пара и жидкости, записи их значений в массив циркулирующих потоков. После расчетов всех совмещенных ступеней определяется невязка покомпонентного материального баланса. При недостаточной точности расчета итерационный процесс повторяется. Метод устойчив и прост при программировании.

Однако при расчете в присутствии водяного пара возможна раскачка итерационного процесса. В этом случае затруднения удается обойти при представлении уравнения теплового баланса и видоизмененного уравнения однократного испарения смеси системой нелинейных уравнений с ограничением на переменные.

Abstract. The combined process of multi-stage condensation and evaporation is a simple and one of the most effective ways of separating a multicomponent mixture. This process can be implemented in a horizontal fractionating apparatus. The coolant (coolant) used at the previous stage of evaporation (condensation), in the direction of travel is sent for reuse in the next stage, etc. This ensures a more complete removal of heat (cold) of the coolant (coolant).

The analysis of the process of combined multi-stage condensation and evaporation of the mixture can be carried out using a graphical combined enthalpy diagram, the equations of the working line and the phase equilibrium curve, as well as on the basis of other patterns used in the analysis of the 
rectification process. Graphic methods are not accurate and are suitable only for analysis of binary mixture separation. Computer calculations based on stable iterative methods are preferred. Due to the essential features of the multi-stage combined condensation and evaporation process, the use of well-known methods for calculating the separation of a multicomponent mixture by distillation is not possible.

In relation to this process, an iterative calculation method has been developed based on mixing steam and liquid flows introduced into a combined stage, solving a system of nonlinear equations of heat, material balances and single evaporation, determining the values of the generated steam and liquid flows, and writing their values to an array of circulating flows. After calculating all the combined steps, the discrepancy of the component-wise material balance is determined. With insufficient accuracy of the calculation, the iterative process is repeated. The method is stable and easy to program.

However, when calculating in the presence of water vapor, an iterative process can build up. In this case, difficulties can be circumvented when presenting the heat balance equation and the modified equation for a single evaporation of a mixture by a system of nonlinear equations with a restriction on variables.

Ключевые слова: многокомпонентная смесь; ректификация; конденсация; испарение; совмещение процессов; теплоподвод; теплоотвод; итерационный расчет

Key words: multicomponent mixture; fractionation; condensation; evaporation; combination of processes; heat input; heat sink; iterative calculation

Совмещенный процесс многоступенчатой конденсации и испарения является простым и, в то же время, одним из эффективных способов разделения многокомпонентной смеси [1]. Данный процесс может быть 
реализован в фракционирующем аппарате горизонтального исполнения. Он характеризуется малыми капитальными вложениями и низкими эксплуатационными затратами.

При эксплуатации аппарата теплоноситель (хладоноситель), использованный на предыдущей ступени испарения (конденсации), по ходу движения направляется для повторного использования в последующую ступень и т.д. Тем самым обеспечивается более полное снятие тепла (холода) теплоносителя (хладоносителя). Причем степень регенерации тепла тем полнее, чем больше разность температур кипения целевых компонентов смеси, выводимых с противоположных концов аппарата. Особенностью процесса является также зависимость эффективности фракционирования от соотношения величин тепла и холода, вводимых в совмещенную ступень разделения [2].

Схема совмещения ступеней конденсации и испарения приведена на рисунке 1 .

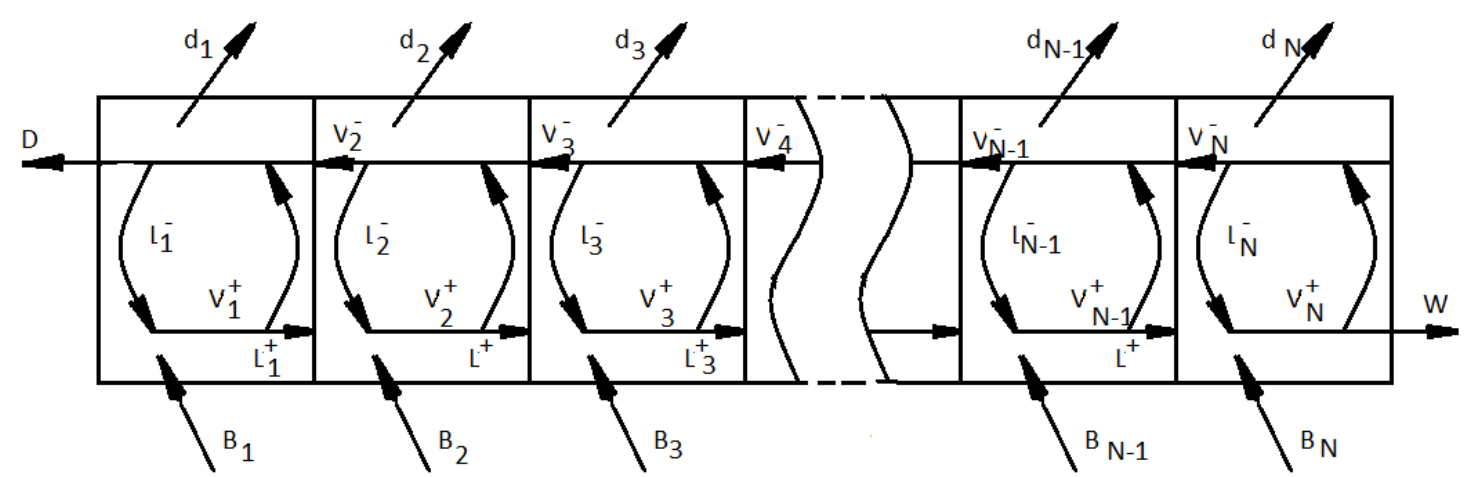

Рисунок 1. Принципиальная схема процесса

В ступень конденсации из соседней ступени вводится поток пара $\mathrm{V}_{\mathrm{j}+1}^{-}$и смешивается с потоком пара $\mathrm{V}_{\mathrm{j}}^{+}$совмещенной ступени испарения. Аналогично в ступень испарения из предыдущей ступени вводится поток жидкости $\mathrm{L}_{\mathrm{j}-1}^{+}$и смешивается с потоком $\mathrm{L}_{\mathrm{j}}^{-}$совмещенной ступени конденсации. Следовательно, некоторое количество продукта циркулирует внутри совмещенной ступени конденсации и испарения. 
Для организации массообмена в ступень конденсации подается холод $\mathrm{d}_{\mathrm{j}}$, в ступень испарения - тепло $\mathrm{B}_{\mathrm{j}}$. В итоге получают высококипящий $\mathrm{W}$ и низкокипящий $\mathrm{D}$ продукты - результат разделения многокомпонентной смеси.

Анализ особенностей процесса можно проводить с помощью уравнений кривой равновесия фаз и рабочей линии ректификации, графической комбинированной энтальпийной диаграммы, а также с использованием иных закономерностей, применяемых при анализе процессов фракционирования. Графические методы не точны и применимы лишь для анализа разделения бинарной смеси. Предпочтительны компьютерные расчеты, основанные на устойчивых итерационных методах.

Ввиду существенной особенности процесса многоступенчатой совмещенной конденсации и испарения применение общеизвестных методов расчета разделения многокомпонентной смеси ректификацией [36] не удается. Применительно к данному процессу нами разработан итерационный метод расчета.

В основу взят метод [7], в котором при моделировании ступени тепломассообменного контакта многокомпонентной смеси решается система нелинейных уравнений теплового, материального балансов и однократного испарения, с определением температуры ступени и доли образованной паровой фазы:

$$
\begin{gathered}
L_{j-1} x_{j-1, i}+V_{j+1} y_{j+1, i}+F_{k} z_{k, i}-L_{j} x_{j i}-V_{j} y_{j i}+F_{j} z_{j i}=0 ; \\
L_{j-1}+V_{j+1}+F_{k}-L_{j}-V_{j}+F_{j}=0 ; \\
Q_{j-1}^{L}+Q_{j+1}^{V}+Q_{k}^{F}-Q_{j}^{L}-Q_{j}^{V}+Q_{j}^{F}+Q_{0}, \quad i=\overline{1, n,} \\
\sum_{i=1}^{n} \frac{z_{j i}}{1+e_{j}\left(K_{j i}-1\right)}=1
\end{gathered}
$$

с учетом ограничения:

$$
0 \leq e_{j} \leq 1, \quad j=1, \ldots, N
$$


где $\mathrm{Z}_{\mathrm{ji}}=\frac{\mathrm{L}_{\mathrm{j}-1, \mathrm{i}}+\mathrm{V}_{\mathrm{j}+1, \mathrm{i}}+\mathrm{F}_{\mathrm{ji}}}{\mathrm{L}_{\mathrm{j}-1}+\mathrm{V}_{\mathrm{j}+1}+\mathrm{F}_{\mathrm{j}}}$.

Уточнение значений потоков и их теплосодержаний проводится по уравнениям:

$$
\begin{gathered}
L_{j}=\left(1-e_{j}\right) \cdot\left(L_{j}+V_{j+1}+F_{j}\right) ; \quad V_{j}=e_{j} \cdot\left(L_{j-1}+V_{j+1}+F_{j}\right) ; \\
h_{j}=\sum L_{j i} \cdot h_{j i} ; \quad H_{j}=\sum V_{j i} \cdot h_{j i} ; \quad L_{j i}=L_{j} \cdot x_{j i} ; \quad V_{j i}=V_{j} \cdot y_{j i},
\end{gathered}
$$

где L, V -расход жидкости и пара;

$\mathrm{F}$-сырьевой поток (боковой погон);

е - доля паровой фазы;

x, y -доля компонента в жидкой и паровой фазах;

z - доля компонента в смеси;

$\mathrm{K}$ - константа фазового равновесия;

h, Н - энтальпии жидкого и парового потоков;

$\mathrm{Q}$ - тепло потока;

$\mathrm{Q}_{0}-$ подвод (отвод) тепла;

n - число компонентов;

$\mathrm{N}$ - число ступеней.

Расчет ступени выполняется итерационно, до выполнения материального и теплового балансов с заданной точностью.

В алгоритме расчета процесса совмещенной многоступенчатой конденсации и испарения система уравнений (1) принимает следующий вид:

для ступени конденсацуии

$$
\begin{gathered}
V_{j+1}^{-} y_{j+1, i}^{-}+V_{j}^{+} y_{j i}^{+}-V_{j}^{-} y_{j i}^{-}-L_{j}^{-} x_{j i}^{-}=0 ; \\
V_{j+1}^{-}+V_{j}^{+}-V_{j}^{-}-L_{j}^{-}=0 ; \\
Q_{j+1}^{V^{-}}+Q_{j}^{V^{+}}-Q_{j}^{V^{-}}-Q_{j}^{L^{-}}-d_{j}=0 .
\end{gathered}
$$

для ступени испарения 


$$
\begin{gathered}
L_{j-1}^{+} x_{j-1, i}^{+}+L_{j}^{-} x_{j i}^{-}-V_{j}^{+} y_{j i}^{+}-L_{j}^{+} x_{j i}^{+}=0 ; \\
L_{j-1}^{+}+L_{j}^{-}-V_{j}^{+}-L_{j}^{+}=0 ; \\
Q_{j-1}^{L^{+}}+Q_{j}^{L^{-}}-Q_{j}^{V^{+}}-Q_{j}^{L^{+}}+B_{j}=0 .
\end{gathered}
$$

Значения потоков $\mathrm{V}_{\mathrm{j}}^{+}$и $\mathrm{L}_{\mathrm{j}}^{-}$выбираются из массива циркулирующих потоков пара и жидкости. Расчет ступеней проводится по вышеприведенному алгоритму, описанному уравнениями (1)-(3). По завершении итерации расчета значения циркулирующих потоков $\mathrm{V}_{j}^{+}$и $\mathrm{L}_{\mathrm{j}}^{-}$ обновляются в соответствующих массивах данных.

После проведения расчета всех ступеней определяют итоговую невязку покомпонентного материального баланса. При невыполнении материального баланса, осуществляется переход к повторному расчету ступеней. В результате итерационного расчета последовательно уточняются составы, расходы и теплосодержания потоков, и минимизируется невязка материального баланса.

При расчетах процессов фракционирования многокомпонентной смеси в присутствии воды возникают затруднения, связанные с раскачкой итерационного вычислительного процесса. В этом случае затруднения удается обойти при иной формулировке задачи [8].

Уравнение теплового баланса и видоизмененное уравнение однократного испарения представляется совместной системой нелинейных уравнений:

$$
\begin{gathered}
L_{j-1} \cdot h_{j-1}+V_{j+1} \cdot H_{j+1}+F_{j} \cdot h_{f}-L_{j} \cdot h_{j}-V_{j} \cdot H_{j}=0, \\
\sum_{i=1}^{n} \frac{z_{j i} \cdot\left(K_{j i}-1\right)}{1+e_{j} \cdot\left(K_{j i}-1\right)}=0
\end{gathered}
$$

с учетом ограничения (3). 
Система уравнений существенно нелинейна. Успешное решение обеспечивает метод Ньютона-Рафсона с расчетом соответствующей матрицы Якоби.

В большинстве случаев на ступенях осуществляется фракционирование парожидкостной смеси, требования ограничения (3) выполняются. Тем не менее, в начале итерационного расчета возможен случай достижения границ ограничения (3). Тогда, в частности, при значительном теплоподводе расчет проводится с наличием на ступени перегретого водяного пара. В итоге возникает раскачка итерационного процесса. Такая ситуация часто возникает при расчетах сложных систем разделения с обратными и рецикловыми потоками. Отличительной особенностью совмещенного процесса многоступенчатого испарения и конденсации смеси является наличие таких потоков. Тепловой баланс ступени при решении задачи (3), (6) с большим вводом тепла не выполняется, температура рассчитанного пара низка, теплосодержание потока мало. Вследствие этого сглаживаются начальные возмущения, уменьшаются колебания.

При достижении другой границы ограничения (3), когда смесь находится в жидкофазном состоянии с температурой ниже температуры ее кипения, задача решается аналогичным образом.

Данная модификация метода позволяет проводить расчеты системы фракционирования любой сложности.

\section{Выводы}

Разработан итерационный метод расчета совмещенного процесса многоступенчатой конденсации и испарения смеси. Метод основан на совместном решении уравнений покомпонентного материального и теплового балансов совместно с уравнением однократного испарения смеси. 
Метод итерационного расчета последовательно уточняет составы, расходы и теплосодержания потоков, минимизирует невязку материального баланса.

\section{Список используемых источников}

1. Умергалин Т.Г. Процесс совмещенной многоступенчатой конденсации и испарения смеси. Уфа: Башкирское книжное издательство, 1991. $150 \mathrm{c}$.

2. Умергалин Т.Г. Оптимизация режима многоступенчатого испарения и конденсации смеси // Нефтепереработка и нефтехимия. 1991. № 8. C. 57-60.

3. Скобло А.И., Молоканов Ю.К., Владимиров А.И. Процессы и аппараты нефтегазопереработки и нефтехимии. М.: РГУ нефти и газа им. И.М. Губкина, 2012. 725 с.

4. Дытнерский Ю.И. Процессы и аппараты химической технологии. М.: Химия, 1995. 324 с.

5. Кондратьев А.А. Расчет ректификации непрерывной смеси в колонне с несколькими вводами питания и отборами // Теоретические основы химической технологии. 1972. Т. 6. № 3. С. 477-479.

6. Александров И.А. Перегонка и ректификация в нефтепереработке. М.: Химия, 1981. 351 с.

7. Умергалин Т.Г., Умергалина Т.В. Расчет тепло-массообмена ступени контакта многокомпонентной смеси // Башкирский химический журнал. 2016. T. 23. № 2. C. 41-43.

8. Умергалин Т.Г. О сходимости расчета процесса ректификации в присутствии насыщенного водяного пара // Теоретические основы химической технологии. 1991. Т. 25. № 2. С. 302-305. 


\section{References}

1. Umergalin T.G. Protsess sovmeshchennoi mnogostupenchatoi kondensatsii $i$ ispareniya smesi [The Process of Combined Multi-Stage Condensation and Evaporation of the Mixture]. Ufa, Bashkirskoe knizhnoe izdatel'stvo Publ., 1991. 150 p. [in Russian].

2. Umergalin T.G. Optimizatsiya rezhima mnogostupenchatogo ispareniya i kondensatsii smesi [Optimization of Multistage Evaporation and Condensation of the Mixture]. Neftepererabotka $i$ neftekhimiya - Oil Processing and Petrochemistry, 1991, No. 8, pp. 57-60. [in Russian].

3. Skoblo A.I., Molokanov Yu.K., Vladimirov A.I. Protsessy $i$ apparaty neftegazopererabotki $i$ neftekhimii [Processes and Apparatus of Oil and Gas Processing and Petrochemistry]. Moscow, RGU nefti i gaza im. I.M. Gubkina Publ., 2012. 725 p. [in Russian].

4. Dytnerskii Yu.I. Protsessy i apparaty khimicheskoi tekhnologii [Processes and Apparatus of Chemical Technology]. Moscow, Khimiya Publ., 1995. 324 p. [in Russian].

5. Kondrat'ev A.A. Raschet rektifikatsii nepreryvnoi smesi v kolonne $\mathrm{s}$ neskol'kimi vvodami pitaniya i otborami [Calculation of Rectification of Continuous Mixture in a Column with Several Feed Inputs and Selections]. Teoreticheskie osnovy khimicheskoi tekhnologii - Theoretical Foundation of Chemical Engineering, 1972, Vol. 6, No. 3. pp. 477-479. [in Russian].

6. Aleksandrov I.A. Peregonka $i$ rektifikatsiya $v$ neftepererabotke [Distillation and Rectification in Oil Refining]. Moscow, Khimiya Publ., 1981. 351 p. [in Russian].

7. Umergalin T.G., Umergalina T.V. Raschet teplo-massoobmena stupeni kontakta mnogokomponentnoi smesi [Calculation of Heat and Mass Transfer for Contact Stage of Multicomponent Mixture]. Bashkirskii khimicheskii zhurnal Bashkir Chemical Journal, 2016, Vol. 23, No. 2, pp. 41-43. [in Russian]. 
8. Umergalin T.G. O skhodimosti rascheta protsessa rektifikatsii v prisutstvii nasyshchennogo vodyanogo para [Convergence of Design Calculations for Rectification in Presence of Saturated Steam]. Teoreticheskie osnovy khimicheskoi tekhnologii - Theoretical Foundation of Chemical Engineering, 1991, Vol. 25, No. 2, pp. 302-305. [in Russian].

\section{Сведения об авторах}

\section{About the author}

Мингалимов Загир Фидратович, аспирант кафедры газохимии и моделирования химико-технологических процессов, УГНТУ, г. Уфа, Российская Федерация

Zagir F. Mingalimov, Post-graduate Student of Gas Chemistry and Methodology of Chemical Engineering Processes Department, USPTU, Ufa, Russian Federation

e-mail: mingalimov.z@gmail.com

Умергалин Талгат Галеевич, д-р техн. наук, профессор кафедры газохимии и моделирования химико-технологических процессов УГНТУ, г. Уфа, Российская Федерация

Talgat G. Umergalin, Doctor of Engineering Sciences, Professor Gas Chemistry and Methodology of Chemical Engineering Processes Department, USPTU, Ufa, Russian Federation

e-mail: umergalin2010@yandex.ru 